\title{
Unlocking the potential of thyroid autoantibodies
}

Two new studies highlight the value of thyroid autoantibodies in predicting both the risk of developing differentiated thyroid cancer (DTC) and the long-term outcome of patients with DTC.

Thyroid autoantibodies are characteristic of Hashimoto thyroiditis, and are associated with lymphocytic infiltration and destruction of the thyroid gland, leading to hypothyroidism. However, the relationship between Hashimoto thyroiditis and DTC is controversial. In particular, debate is ongoing as to whether the inflammation in Hashimoto thyroiditis is a cause or consequence of DTC. Additionally, the effect of inflammation on the outcome of patients with DTC is uncertain.

In the first study, researchers led by Juan Carlos Jaume analysed the medical records of 2,811 patients referred for thyroid surgery, of whom 582 had a pathology-confirmed diagnosis of Hashimoto thyroiditis. 365 patients with Hashimoto thyroiditis were euthyroid; the hypothyroid patients were stratified by the replacement dose of levothyroxine administered (low, middle or high). Overall, the relative risk of DTC was significantly increased only in euthyroid patients with Hashimoto thyroiditis and hypothyroid patients with Hashimoto thyroiditis on low-dose levothyroxine. Hashimoto thyroiditis thus increases the risk of DTC, but only in patients with functional or partially functional thyroid glands. The elevated risk of DTC in these groups was reduced in patients with high serum titres of thyroid peroxidase autoantibodies (TPOAb), suggesting that high TPOAb titres protect against DTC in patients with Hashimoto thyroiditis.

In the second study, Cosimo Durante and colleagues investigated the possible association between thyroglobulin autoantibodies (TgAb) and the outcome of patients with papillary thyroid cancer (PTC) - the most common type of DTC. The researchers analysed data from 220 TgAb-positive and 1,020 TgAb-negative patients with PTC who had undergone thyroidectomy with or without radioiodine remnant ablation. TgAb-positive status was associated with more aggressive disease at presentation and worse longterm outcomes, including higher rates of both persistent and recurrent disease than in TgAb-negative patients. At the 1-year follow-up, patients who had regained TgAb-negative status were threefold less

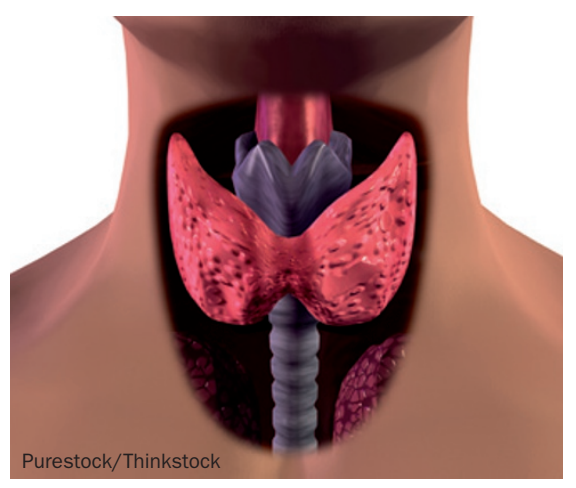

likely to have persistent and/or recurrent disease than those patients whose titres were still positive, suggesting a protective effect of TgAb-negative status.

These studies demonstrate the predictive and prognostic value of specific Hashimoto thyroiditis autoantibodies in DTC, warranting further research on the role of inflammation in DTC.

\section{David Holmes}

Original articles Paparodis, R. et al. Hashimoto
thyroiditis pathology and risk for thyroid cancer. Thyroid
doi:10.1089/thy.2013.0588 | Durante, C. et al. Clinical
aggressiveness and long-term outcome in patients with
papillary thyroid cancer and circulating anti-thyroglobulin
autoantibodies. Thyroid doi:10.1089/thy.2013.0698

